\title{
Bayesian small-area modelling of COVID-19 cases and deaths in England and association with key risk factors: a combined space-time Susceptible- Exposed-Infected-Removed (SEIR) model
}

\section{Benn Sartorius ( $\sim$ benn.sartorius1@lshtm.ac.uk)}

London School of Hygiene and Tropical Medicine https://orcid.org/0000-0001-6761-2325

Andrew Lawson

Medical University of South Carolina

Rachel L. Pullan

London School of Hygiene and Tropical Medicine

\section{Research Article}

Keywords: COVID-19, SARS-CoV-2, small-area Bayesian estimation, Susceptible-Exposed-InfectedRemoved (SEIR), mobility, non-pharmaceutical intervention, Middle Layer Super Output Area, England

Posted Date: November 4th, 2020

DOl: https://doi.org/10.21203/rs.3.rs-102191/v1

License: (c) (i) This work is licensed under a Creative Commons Attribution 4.0 International License.

Read Full License 
2 Bayesian small-area modelling of COVID-19 cases and deaths in England and association with key risk factors: a combined space-time Susceptible-Exposed-Infected-Removed (SEIR) model

4 Sartorius $\mathrm{B}^{1 *}$, Lawson $\mathrm{AB}^{2}$, Pullan $\mathrm{RL}^{1}$

51 Department of Disease Control, Faculty of Tropical and Infectious Diseases, London School of

$6 \quad$ Hygiene \& Tropical Medicine, Keppel Street, London, WC1E 7HT, UK

72 Division of Biostatistics and Bioinformatics, Department of Public Health Sciences, Medical

8 University of South Carolina, Columbia, SC 29208, United States

9

10 *Correspondence: Dr Benn Sartorius; Address: Keppel St, Department of Disease Control, Faculty of

11 Infectious and Tropical Diseases, London School of Hygiene \& Tropical Medicine, Bloomsbury,

12 London WC1E 7HT, United Kingdom; Email: benn.sartorius1@Ishtm.ac.uk. 
Background: COVID-19 caseloads in England appear have passed through a first peak, with evidence of an emerging second wave. To ensure continued response to the epidemic is most effective, it is imperative to better understand both retrospectively and prospectively the geographical evolution of COVID-19 caseloads and deaths, identify localised areas in space-time at significantly higher risk, quantify the impact of changes in localised population mobility (or movement) on caseloads, identify localised risk factors for increased mortality and project the likely course of the epidemic at smallarea resolution in coming weeks.

Methods: We applied a Bayesian space-time SEIR model to assess the spatiotemporal variability of COVID-19 caseloads (transmission) and deaths at small-area scale in England (Middle Layer Super Output Area [MSOA], 6791 units) and by week (using observed data from week 5 to 34), including key determinants, the modelled transmission dynamics and spatial-temporal random effects. We also estimate the number of cases and deaths at small-area resolution with uncertainty projected forward in time by MSOA (up to week 51 of 2020), the impact mobility reductions (and subsequent easing) have had on COVID-19 caseloads and quantify the impact of key socio-demographic risk factors on COVID-19 related mortality risk by MSOA.

Results: Reductions in population mobility due the course of the first lockdown had a significant impact on the reduction of COVID-19 caseloads across England, however local authorities have had a varied rate of reduction in population movement which our model suggest has substantially impacted the geographic heterogeneity in caseloads at small-area scale. The steady gain in population mobility, observed from late April, appears to have contributed to a slowdown in caseload reductions towards late June and subsequent steady increase signalling the start of the second wave. MSOA with higher proportions of elderly ( $70+$ years of age) and elderly living in 
deprivation, both with very distinct geographic distributions, have a significantly elevated COVID-19 mortality rates.

Conclusions: While non-pharmaceutical interventions (that is, reductions in population mobility and social distancing) had a profound impact on the trajectory of the first wave of the COVID-19 outbreak in England, increased population mobility appears to have contributed to the current increase signalling the start of the second wave. A number of contiguous small-areas appear to be at a significant elevated risk of high COVID-19 transmission, many of which are also at increased risk for higher mortality rates. A geographically staggered re-introduction of intensified social distancing measures is advised and limited cross MSOA movement if the magnitude and geographic extent of the second wave is to be reduced.

Keywords: COVID-19, SARS-CoV-2, small-area Bayesian estimation, Susceptible-Exposed-InfectedRemoved (SEIR), mobility, non-pharmaceutical intervention, Middle Layer Super Output Area, England

\section{Introduction}

The first cases of COVID-19 in the United Kingdom were confirmed on 31 January $2020^{1}$.

Despite implementation of non-pharmaceutical interventions, including closure of non-essential services and subsequent and stay at home orders and school closures on 20 and 23 March 2020 respectively, this was not sufficient to contain the first wave of the UK outbreak, although these measures did serve to slow early transmission ${ }^{2}$. The full extent of the effect of the mobility restrictions on COVID-19 transmission has not been fully elucidated nor explicitly quantified ${ }^{3,4}$. In addition, the attributability of hypothesised socio-demographic risk factors for COVID-19 related mortality such as deprivation, crowding and ethnicity have also not been fully quantified nor unpacked at small-area resolution. 
67 As COVID-19 caseloads in England appear to rising towards a second peak (wave), to ensure continued response to the epidemic is most effective, it is imperative that we better understand, both retrospectively and prospectively, the geographical evolution of COVID-19 and localised areas in space-time at higher risk of severe disease burden and mortality. Assessing the impact of mobility and population-density (crowding) on differences in caseloads at small area scale will not only allow assessment of the impact that social distance measures have had on the magnitude and timing of the waves, but also allow counterfactual assessment of what this magnitude may have been in the absence of these non-pharmaceutical interventions. More importantly as we enter the second wave with increased likelihood of local outbreaks, this work will help identify small-areas at elevated risk of transmission and mortality and subsequently inform when it might be safe to start lifting social distancing measures at small-area scale in a geographic staggered approach. Furthermore, a better understanding of the spatiotemporal dynamics of this COVID-19 outbreak, and accurate characterisation of the likely spread and magnitude, will be critical for the design of timely and costeffective control strategies to minimise the spread of future such pandemics, and to help establish better early warning systems ${ }^{5-7}$.

We apply a stochastic Susceptible-Exposed-Infected-Removed (SEIR) space-time model for publicly available COVID-19 confirmed case and death data in England at small area scale and by week to map the likely trajectory, peak and duration of the outbreak at MSOA level by week, quantifying the impact of mobility. We utilise weekly MSOA level population mobility data against observed confirmed COVID-19 case data to assess the impact mobility reduction at small-area scale has had on case transmission, and counterfactually what the magnitude may have been under the scenario of no mobility loss. Furthermore, the inclusion of the death compartment of the model using observed weekly COVID-19 related deaths at MSOA level and linkage to key socio-demographic risk factors allows this framework to identify not only small-areas with significantly elevated mortality risk but 
also what localised contextual factors may be significantly contributing to his. We apply an extension of the basic model ${ }^{8}$ by combining the SEIR implementation with a spatial conditional autoregressive (CAR) model, and also extend this implementation by considering neighbourhood infection effects for the infection process ${ }^{9}$. The space-time dynamics of COVID-19 related mortality in England and associated risk factors/determinants at MSOA level are also assessed to identify vulnerable MSOA.

\section{Results}

Observed spatial patterns for COVID-19 caseloads at MSOA: A spatial assessment of COVID-19 caseloads (scaled by population totals) for the observed data input period (week 9 to 34 of 2020), suggests a non-random distribution to high incidence MSOA across England with distinct pockets of high case rates centred in and around metropolitan areas (Figure 1a) for example Leicester, Birmingham, Liverpool, Manchester etc. The analysis of localised spatial clustering identified significant hot spots (at MSOA level) in Bedford/Bedfordshire, Leicester, Peterborough, large contiguous band in Cheshire and Merseyside, another contiguous band spanning Leeds-WakefieldBarnsley-Sheffield and in the north Newcastle-Sunderland (Figure 1b). Additional pockets of significant excess COVID-19 case rates in less metropolitan type areas were identified in pockets throughout England, for example Ashford, Kings Lynn and West Norfolk and Burrow-in-Furness.

Spatial temporal patterns in population mobility at MSOA: Despite an overall significant reduction in population mobility from early March (week 9) to mid-April (week 14) of 2020 (Figure 1c), mobility rates began to increase from week 16 and median mobility levels across MSOA had been almost attained pre-lock March/April down levels by week 34 of 2020. The rate of cumulative mobility loss by mid-April varied across MSOA (Figure 1d), with lower levels of mobility loss generally in part of West and East Midlands, Northwest, York and the Humber land as well as North East England. A large band of significant hotspots for lower cumulative mobility loss at week 14 were identified in a large contiguous aggregation of MSOA spanning from Birmingham through to Leicester, Nottingham, 
117 Sheffield, Leeds, Manchester and Liverpool (Figure 1e). Furthermore, significant clustering of lower

118 cumulative mobility loss was also observed in Newcastle and surrounds, Norwich and coastline areas

119 immediately to the east of Norwich, Tendering/Colchester, Canterbury/Dover and west of

120 Southampton for example. 
a)

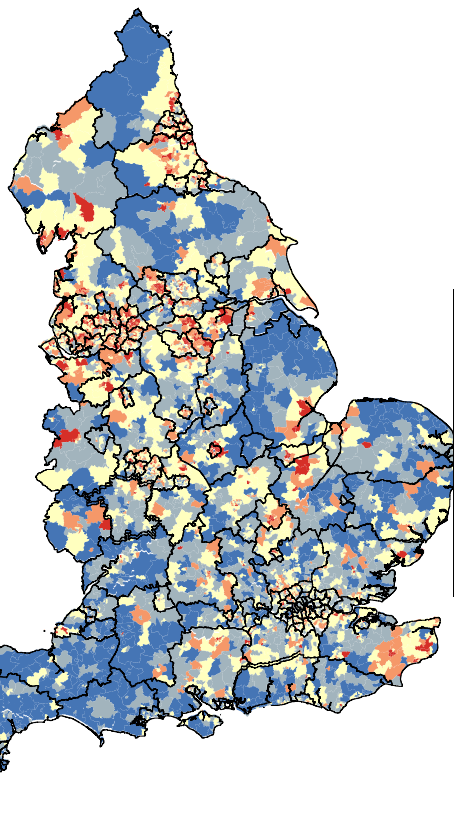

122

Figure 1: a) cumulative case rates at week 34 at English MSOA level ( $N=6791)$; b) statistically significant localised hot and cold spots for higher and lower cumulative COVID-19 cases at MSOA resolution at week 34 of 2020 using the Getis-Ord Gi* localised clustering statistic; c) Box plot of mobility by MSOA and week ; d) cumulative mobility loss (darker red indicates lower mobility loss) by MSOA in England ( $n=6791$ ) from week 9 to 14 of 2020; e) statistically significant localised hot and cold spots for higher and lower cumulative mobility loss at MSOA resolution from week 9 to 14 of 2020 using the GetisOrd Gi* statistic
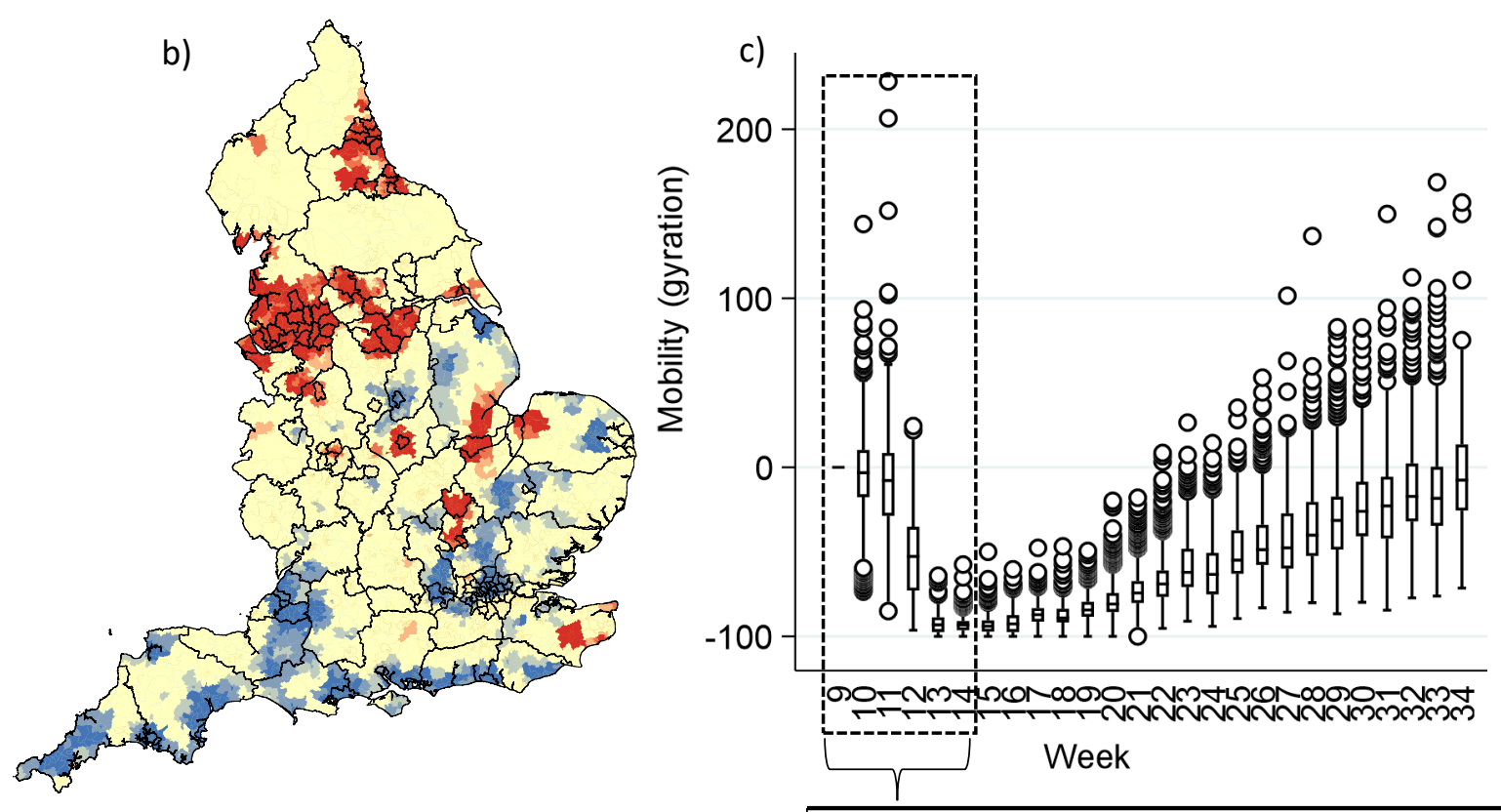

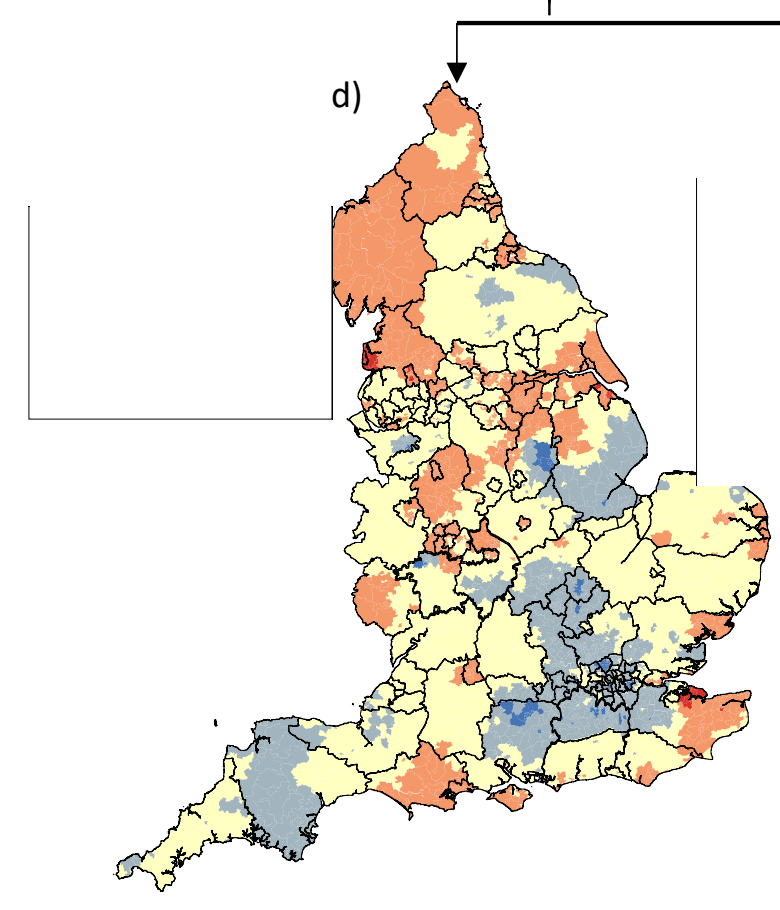

e)

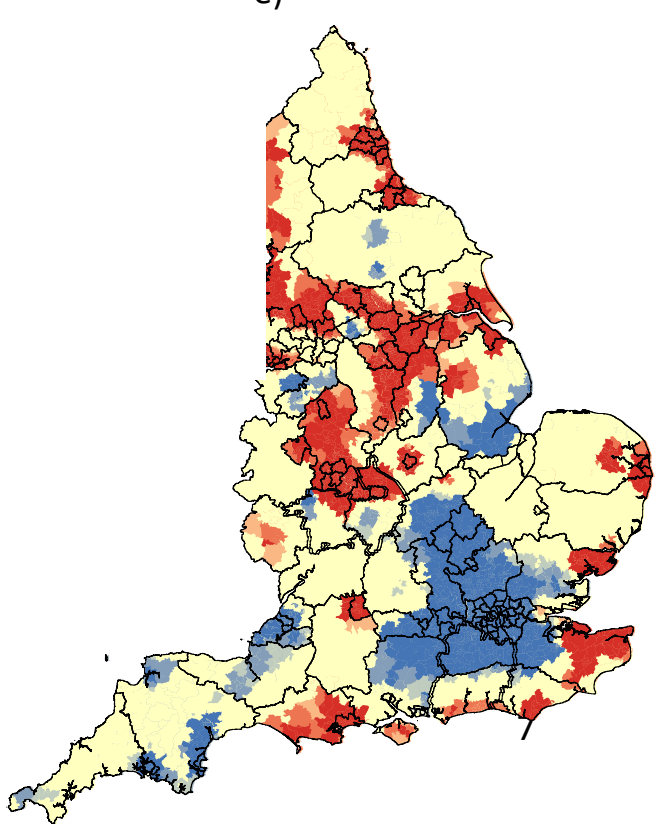


Spatial-temporal patterns for COVID-19 mortality: The highest cumulative death rates for COVID-19 at MSOA resolution from week 9 to 26 of 2020 were observed in parts of North London, most of Birmingham and immediate surrounding MSOA, a large area spanning Cheshire-Merseyside, and lastly in and surrounding Newcastle (Figure 2a). Significant hotspots for COVID-19 related mortality were highly clustered and were identified in contiguous MSOA located in North London (EalingBrent-Hillingdon-Harrow-Barnet-Enfield-Three Rivers-Hertsmere-), West Midlands (BirminghamSandwell-Walsall-Dudley-Wolverhampton), North West (Liverpool-Manchester-Cheshire), Sheffield, and Newcastle (County Durham-Sunderland-Gateshead) (Figure 2b). The two principle risk factors associated with COVID-19 mortality risk and leveraged in this study, namely proportion of MSOA population aged 70 years or older and elderly population living in deprivation have two very distinct spatial distributions across England (Figure 2c and e respectively). The MSOA with higher proportions of population aged 70 years or older appear significantly clustered along the East of England coastline and the coastline in Southwest and South East regions of England (Figure 2d). Additional significant hot spots for elderly population clustering were identified outside key metropolitan centres, for example: west of Birmingham, north of Liverpool, south of Manchester, north of Nottingham and north of Bradford-Leeds (Figure 2d).

Spatial patterns for deprivation: The most deprived (worst 10 percentile) neighbourhoods (680 MSOA) are not evenly distributed across England (Figure 2e, f), with $50 \%$ of these areas located within just 20 local authority districts, notably - Birmingham with 62 such neighbourhoods; Liverpool, 35; Leeds and Manchester, 29 each, and Bradford, 20. Significant spatial clustering of the most deprived neighbourhoods is concentrated in large areas of contiguous MSOA located in London, West Midlands, Northwest, Yorkshire and Northeast England, with pockets of significant deprivation surrounded by less/least deprived neighbourhoods (Figure 2f). Furthermore, concentrations of deprivation among these $680 \mathrm{MSOA}$ are also disproportionately concentrated in in large urban conurbations (142 Industrial and Multi-ethnic, 125 Larger Towns and Cities; 80 Ethnically Diverse Metropolitan Living; 33 urban living), as well as in areas that are (or historically were) 

classified as heavy industry manufacturing and/or mining sectors (74 Manufacturing legacy, 42

155 service economy; 39 mining legacy). A statistical assessment of spatial clustering for elderly

156 population living in deprivation confirms the significant concentration of hot spots in the

157 metropolitans, namely: London, Birmingham, Liverpool, Manchester, Nottingham, Sheffield-Leeds

158 and Newcastle (Figure 2f). 
a)

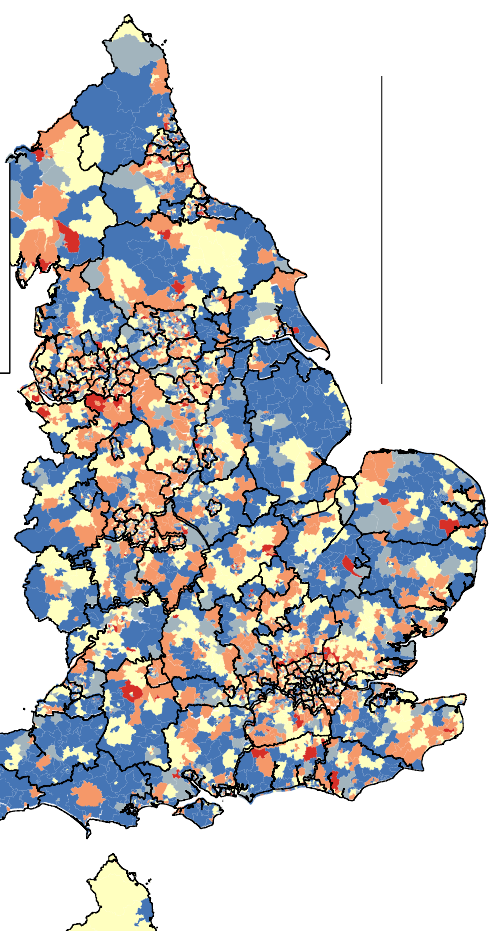

161

162
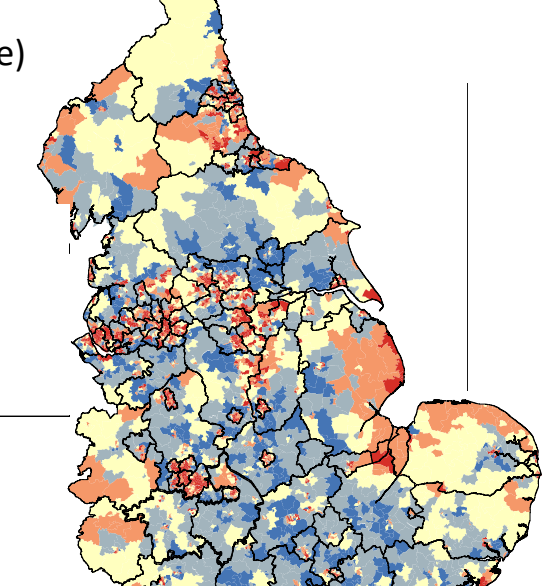

166

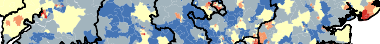

(1) $1,13 x$



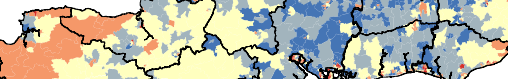

b)

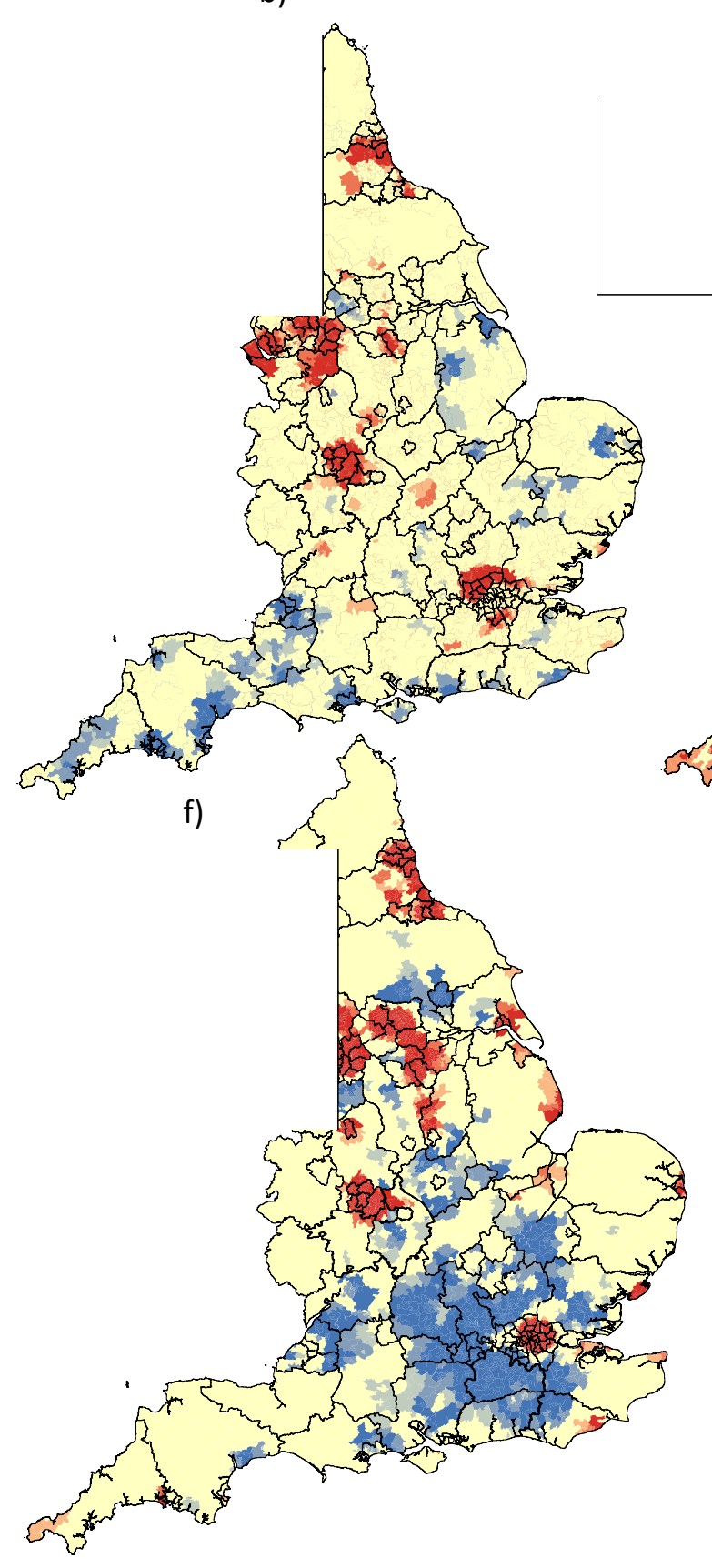

c)

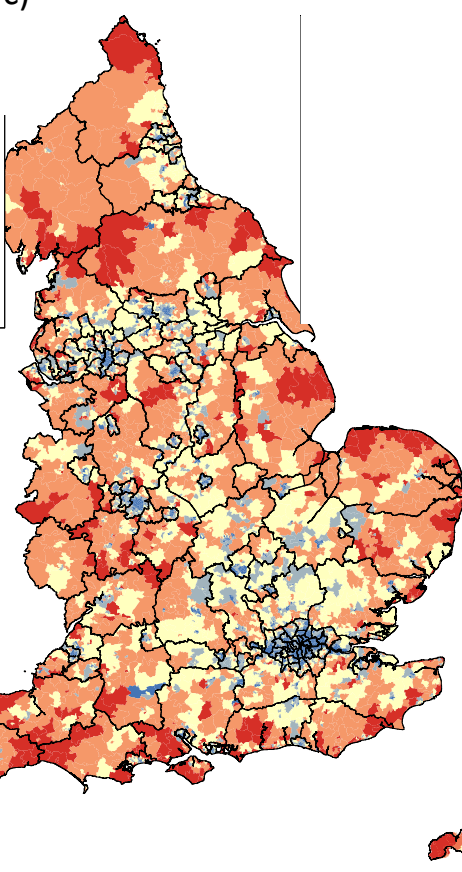

d)

)

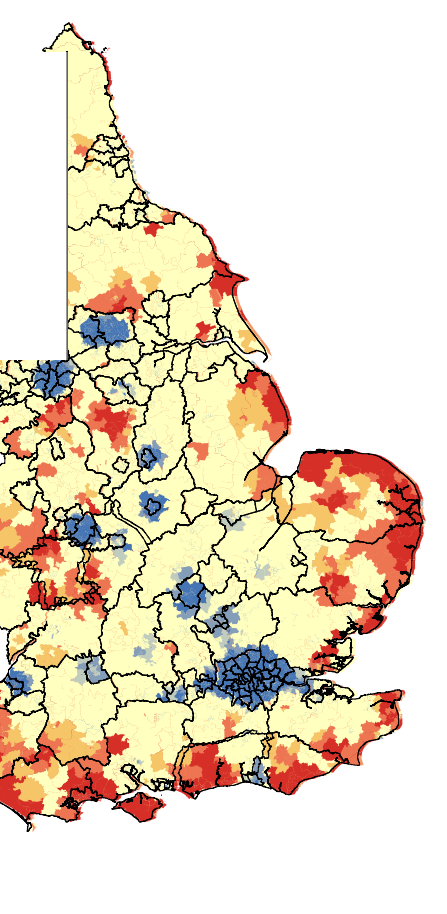

Figure 2: a) Cumulative case rates at week 34 at English MSOA level $(\mathrm{N}=6791) ; \mathrm{b})$ statistically significant localised hot and cold spots for higher and lower cumulative COVID-19 cases at MSOA resolution at week 34 of 2020 using the Getis-Ord Gi* statistic; c) proportion of population aged 70+ at MSOA resolution; d) statistically significant localised hot and cold spots for higher and lower proportion of population aged 70+ at MSOA resolution using the Getis-Ord Gi* statistic; e) Index of multiple deprivation at MSOA resolution; f) statistically significant localised hot and cold spots for higher and lower proportion of IMD at MSOA resolution using the Getis-Ord Gi* statistic. 
Space-time SEIR dynamics: The full multivariate Bayesian space-time model estimated an asymptomatic fraction of 0.2 (95\% UI: 0.06-0.39) i.e. for every 5 confirmed cases there is likely to be 1 additional asymptomatic case (Table 2). A decrease in mobility at MSOA scale has had a significant reduction on caseloads i.e. each unit increase in cumulative mobility (rescaled to be between 0 and 1) appears to be associated with a +3.6-unit increase in cases on the log scale (95\%UI: 3.57-3.61)

172 (Table 1). The change in cumulative mobility also appears to significantly interact with the natural

173 logarithm of population density within a MSOA i.e. the impact of higher mobility is amplified by

174 increasing population density $(\beta=+0.05)$. Increasingly proportion of a MSOA population aged 70

175 years or older was strongly and significantly associated with increased COVID-19 related deaths

$176(\beta=+4.86,95 \% \mathrm{Cl}: 4.59-5.15)$ while a decreasing proportion of the elderly population in a given

177 MSOA living in deprivation appears to have a strong and significant negative association $(\beta=-0.25$, 95\% Cl: -0.34, -0.10) (Table 1).

Table 1: Posterior statistics from the Bayesian multivariable space-time SEIR model using WinBUGS 180

\begin{tabular}{|c|c|c|c|c|}
\hline Compartment & Node & Coef. $(\beta)$ & $2.50 \%$ & $97.50 \%$ \\
\hline \multirow{5}{*}{ Cases } & Asymptomatic fraction & +0.20 & +0.06 & +0.39 \\
\hline & Cumulative mobility loss & +3.59 & +3.57 & +3.61 \\
\hline & Cumulative mobility loss $X \ln$ (Population per $\mathrm{km}^{2}$ ) & +0.05 & +0.05 & +0.05 \\
\hline & $\tau\left(v_{1 i}\right)$-spatially structured random effect & 2.35 & 2.05 & 2.70 \\
\hline & $\tau\left(\cup_{1 i}\right)$-unstructured random effect & 14.53 & 12.06 & 17.47 \\
\hline \multirow{4}{*}{ Deaths } & Proportion aged 70 years and older & +4.86 & +4.59 & +5.15 \\
\hline & Elderly population living in material deprivation index ${ }^{i}$ & -0.25 & -0.34 & -0.10 \\
\hline & $\tau\left(v_{2 i}\right)$ - spatially structured random effect & 0.81 & 0.73 & 0.88 \\
\hline & $\tau\left(U 2_{i}\right)-$ unstructured random effect & 1.44 & 1.34 & 1.55 \\
\hline
\end{tabular}

181 i: increasing score indicate lower level of material deprivation.

182 Posterior space-time projections from ST-SEIR model: The posterior projections from the full space-

183 time SEIR model closely fit with the observed temporal course of case and deaths for COVID-19 over

184 the observed data input period (spanning the first wave), namely week 9 to 26 for deaths and week

1859 to 34 for cases (Figure 3). From week 27 the model fairly closely tracks the increase in caseloads which appears to signal the start of the second wave. Post week 34 i.e. the model out of prediction 
phase, we note an exponential rise in observed cases beginning around week 35 . The model prediction based on averted cases from lockdown in the first phase combined with the impact of steadily increasing population mobility appears to fairly accurately track the exponential rise from week 35 and suggests a likely second wave peak of almost 45,000 cases per week by week 43/44 (i.e. latter half of October) of this year (Figure 3). Secondly, the model prediction based on the impact of observed mobility change alone, without accounting for the averted (or counterfactual) caseloads in the first wave, appears to significantly underestimate the trajectory of the outbreak in the current phase post week 34 (Figure 3). Interestingly while the death compartment of the model closely fits significantly overestimate the death rate observed thus far in the second wave (Figure 3).

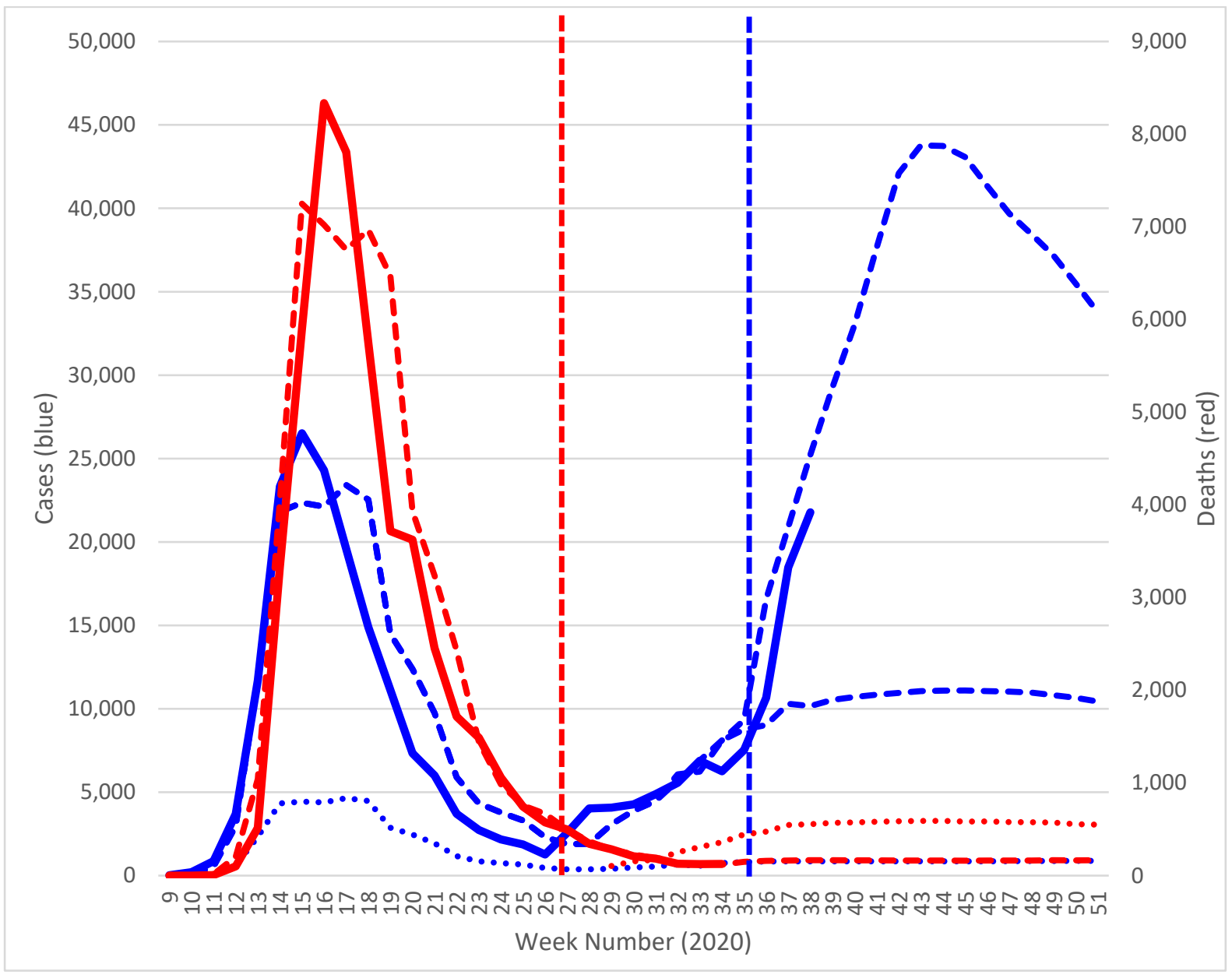

Figure 3: Observed and model fitted number of confirmed cases and deaths in England from week 9 to 34 of 2020, with projections to week 51 of 2020. Vertical coloured line represents the truncation 
201 point for observed input data for cases (week 34$)$ and deaths (week 26) respectively. Blue solid line $=$ observed number of weekly COVID-19 cases; blue dashed line = model fitted cases; dotted blue line = asymptomatic cases; red solid line = observed number of weekly COVID-19 deaths, red dashed line= model fitted deaths; red dotted line $=$ COVID-19 deaths based on mortality rate from first wave. 
Model predicted hotspots for weeks 35-40: The forward projection of the model for weeks 35 to 40 at MSOA level suggests high and significant case rates forecast for large contiguous area spanning North West and Yorkshire regions (Liverpool-Manchester complex, contiguous band spanning Bradford-Calderdale-Kirklees-Leeds-Wakefield-Barnsley-Doncaster-Rotherham-Sheffield, contiguous band of MSOA in and around Kingston upon Hull), large band of connected MSOA in the north east spanning from Newcastle through to Stockton-On-Tees, high risk pockets through the midlands (Stoke-on-Trent, Wolverhampton-Walsall-Dudley-Sandwell, Leicester, Peterborough) and two additional pockets in southern England (Swindon, Ashford-Canterbury-Thanet) (Figure 4a/c). The

214 forecast pockets for significantly high COVID-19 related mortality are more focused/smaller in 215 geographic extent compared to the caseloads with some overlap between areas with project high 216 caseloads and high death rates: Blackpool, Manchester, band spanning Bradford-Leeds-Kirklees-

217 Wakefield, South Tyneside-Sunderland, large band spanning Wolverhampton-Birmingham-Coventry, 218 Leicester and Swindon (Figure 4b/d). Additional pockets of high mortality rate risk but with lowmoderate projected caseload rates were identified, with multiple pockets in the south of England (Wiltshire, North Devon, Cornwall, South Somerset-Dorset and Swale). 

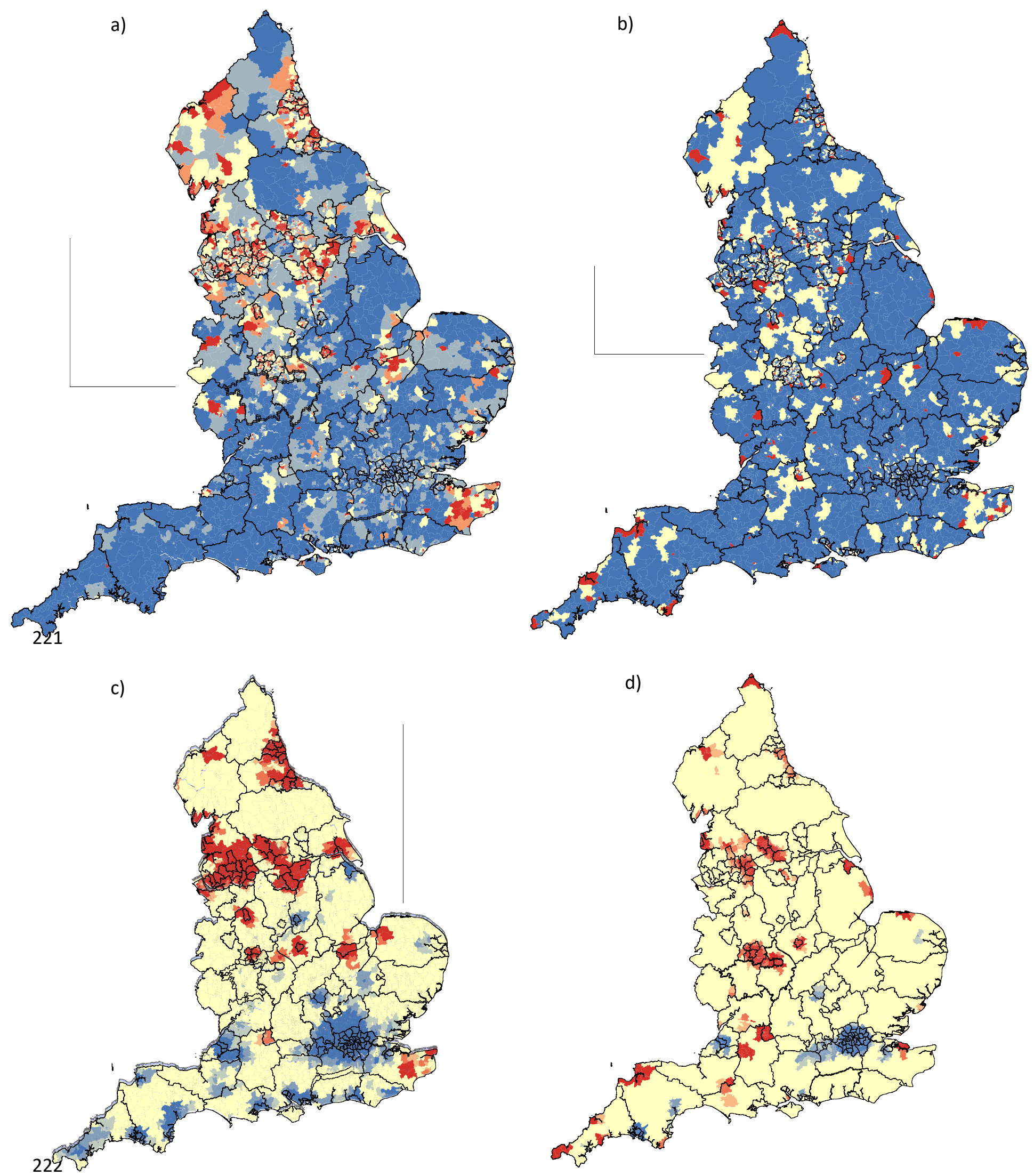
Figure 4: Model projected COVID case (a) and death (b) rates per 100,000 at MSOA level for week 34 to 40 of 2020; statistically significant localised hot and cold spots for COVID-19 case (c) and death (d) rates at MSOA level for week 34 to 40, 2020

\section{Discussion}

To both support and respond effectively to surveillance activities such as NHS Test and Trace or universal weekly testing ${ }^{10}$, it is essential to unpack the spatial-temporal dynamics of the COVID-19 outbreak and related mortality at high spatial resolution (e.g. MSOA) over time and assess the contribution of key risk factors to differences in risk across space and time. Our high resolution spatial-temporal (MSOA by week) model suggest that social distancing measures, as estimated by the impact on population mobility patterns, have had a significant impact on the reduction of COVID-19 caseloads across England, in line with previous studies, for example in China and Italy ${ }^{11-13}$. However, local authorities have seen varied rates of reduction of population movement, significantly impacting the cumulative caseloads at small-area scale. A sensitivity analysis whereby mobility restrictions are eased at various intensities at MSOA scale suggests that a steady gain in mobility, as observed from late April, may have contributed to a slowdown in caseload reductions towards late June and subsequent steady rise thereafter until late August (Figure 3). Subsequent to that the model prediction and observed caseloads data suggest an exponential rise from week 35 (early September). As such, it would appear that a critical tipping point has been reached, and the model suggests particular groupings of MSOA at significant increased risk of future caseloads and deaths. Further targeted implementation of local lockdown is essential at this stage. A geographically staggered approach combined with enhanced community surveillance ${ }^{10}$ will become increasingly important if we are to reduce the magnitude of the second wave in England and plan for subsequent waves/cycles in the absence of an effective vaccine.

Crucially, our results highlight differences in epidemic dynamics across small areas in England, emphasising the importance of monitoring at fine sub-national scale. Our findings suggest that a 
significant proportion of caseload and death variance is spatially structured, and that caseloads in neighbouring local authorities of a given MSOA in the preceding time point are significantly associated with caseloads in current time point. This - coupled with the large significant regional clustering of high caseloads observed - has important implications for informing limitations on movement between areas in the subsequent phases of lockdown easing. Small-area lockdowns could help reduce COVID-19 transmission while minimizing the size of the population (and economy) that needs to be disrupted to continue to release reduced transmission. Furthermore, deaths due to COVID-19 appear to be significantly clustered in space, with some overlap between areas in and around metropolitan centres with higher levels of elderly population living in deprivation (prominent examples: Wolverhampton-Birmingham complex, Blackpool, Manchester, Bradford-Leeds-Wakefield complex and south of Newcastle). However additional pockets of excess mortality risk were also identified in areas in less deprived coastal areas, however from our analyses many of these localities have much higher proportions of elderly population which may in part explain these clusters.

Human mobility has been demonstrated to play an important role in the early stages of the COVID19 epidemic ${ }^{11}$ and in particular reductions observed in digital data sources of mobility appear to correlate well with incidence ${ }^{12}$ as well as contact patterns ${ }^{14}$. As many countries continue to bring their COVID-19 outbreaks under control, evidence on the effectiveness of current lockdowns continues to emerge ${ }^{15-18}$. Social distancing measures have had a significant impact on the reduction of COVID-19 caseloads across England, however local authorities have had a varied rate of reduction of population movement, which has substantially impacted the cumulative caseloads at small-area scale. While non-pharmaceutical interventions such as social distancing have had a profound impact on the trajectory of the COVID-19 outbreak in England, country wide relaxing of these measures from June 2020 and gradual observed increases in population mobility which started even prior to this, risks a rebound in a number of local authorities which still have caseloads above the threshold required to realise MSOA cessation. 
This study is subject to limitations. First, confirmed COVID-19 cases were linked to the MSOA where the test was carried out, rather than patient residence. This is of greater concern for metropolitan areas, where a substantial proportion of patients may have been admitted or transferred to neighbouring trusts before being tested. Secondly, the data are also vulnerable to bias introduced by variable testing rates between Trusts, which may have contributed to greater between MSOA variability. Thirdly, we are assessing the association between aggregated cases/deaths at MSOA level with aggregated risk factor variables at the same level. Thus we cannot discount the possible impact of ecological fallacy in the observed association. A final limitation relates to the mobility data and lack of detail regarding movement between local authorities. While we have demonstrated the importance of mobility loss within a given MSOA on its subsequent caseloads, the impact of movement between local authorities (and density thereof) would provide additional important information regarding cross locality mixing and further targeted restriction of movement between particular higher risk areas.

\section{Conclusions}

This study highlights the importance and usefulness of space-time framework to unpack the dynamics of the COVID-19 outbreak at high resolution (MSOA) and in time, identify particular smallareas at elevated risk of transmission as well as COVID-19 related mortality. Furthermore, at small area scale we demonstrated the utility of longitudinal mobility data for real-time surveillance of the impact of social distancing interventions, and thereby can also allow national authorities to assess the impact on outbreak dynamics at small-area. It is advisable to pre-emptively assess what impact changes in mobility post lockdown easing will have on outbreak rebound potential at localised geographic scale, as well as to include monitoring changes in daily/weekly mobility patterns. Lastly areas with high proportions of elderly population and in particularly elderly population living in deprivation are at significantly greater risk of COVID-19 related mortality, and therefore enhanced surveillance/case follow-up should be ensured in these areas. 


\section{Overview}

300

This analysis adheres to the Guidelines for Accurate and Transparent Health Estimates Reporting

301 standards (GATHER) ${ }^{19}$ (Supplementary Material Section 1). The data used are all in the public domain. Data processing and analyses were performed using Stata 16, and WINBUGS. The statistical code for implementing the Bayesian model in WINBUGS is provided in Supplementary Material

304 (Supplementary Material Section 2).

Data

A summary of the input data and sources is presented in Table 2 . The weekly number of confirmed

COVID-19 cases (Pillar 1 - Government's mass testing programme) and deaths for each MSOA Middle dashboard (https://coronavirus.data.gov.uk/) for the period of February 24, 2020 (week 5) to August 23, 2020 (week 34). The MSOA shape file, used to create the adjacency matrix for the model below and for mapping of the model posteriors, was obtained from the United Kingdom government

312 geoportal platform: https://geoportal.statistics.gov.uk/datasets/middle-layer-super-output-areas-

313 december-2011-boundaries-ew-bfc-1. Weekly COVID-19 deaths at MSOA resolution were also extracted from the NHS PHE Dashboard (https://coronavirus.data.gov.uk/).

Table 2: List of model components, relevant variables and data sources

\begin{tabular}{|c|c|c|c|c|c|}
\hline $\begin{array}{l}\text { Model } \\
\text { component }\end{array}$ & Variable & Source (open access unless indicated*) & $\begin{array}{l}\text { Spatial } \\
\text { resolution }\end{array}$ & $\begin{array}{l}\text { Temporal } \\
\text { resolution }\end{array}$ & Compartment \\
\hline
\end{tabular}




\begin{tabular}{|c|c|c|c|c|c|}
\hline & & $\begin{array}{l}\text { of death or reporting date (depending on } \\
\text { availability). Deaths are allocated to the } \\
\text { deceased's usual area of residence. }\end{array}$ & & & \\
\hline $\begin{array}{l}\text { Covariate } \\
\text { (daily } \\
\text { varying) }\end{array}$ & $\begin{array}{l}\text { Mobility (population } \\
\text { movement index) }\end{array}$ & $\begin{array}{l}\text { Oxford COVID-19 impact monitor (Cuebiq) } \\
\text { (https://oxford-covid-19.com/) }\end{array}$ & MSOA & Daily & Cases \\
\hline \multirow{4}{*}{$\begin{array}{l}\text { Covariates } \\
\text { (annual } \\
\text { estimates, } \\
\text { weekly } \\
\text { fixed) }\end{array}$} & $\begin{array}{l}\text { Age, ethnic } \\
\text { structure }\end{array}$ & ONS (https://www.ons.gov.uk/) & MSOA & 2020 & Deaths \\
\hline & Population density & ONS (https://www.ons.gov.uk/) & MSOA & 2020 & Cases \\
\hline & $\begin{array}{l}\text { Index of Multiple } \\
\text { Deprivation (IMD) }\end{array}$ & $\begin{array}{l}\text { Multiple deprivation experienced by Lower } \\
\text { Layer Super Output Area (LSOA) in England } \\
\text { (https://www.gov.uk/government/statistics/engli } \\
\text { sh-indices-of-deprivation-2019) }\end{array}$ & MSOA & 2019 & Deaths \\
\hline & $\begin{array}{l}\text { Elderly population } \\
\text { proportion living in } \\
\text { deprivation }\end{array}$ & $\begin{array}{l}\text { Public Health England - Local Health: } \\
\text { https://www.localhealth.org.uk/ }\end{array}$ & MPSA & 2019 & Deaths \\
\hline
\end{tabular}

316 Data on population size, population density and the proportion of the population aged 70 years and

317 older as well as proportion of population that is of Black or mixed ethnicity by MSOA were obtained

318 from the Office for National Statistics (ONS) (https://www.ons.gov.uk/). Furthermore, we also

319 extracted the Index of Multiple Deprivation (IMD) score and proportion of the elderly population

320 living in deprivation for each MSOA in England for 2019 (Public Health England: Local Health.

321 [Accessed: 24/08/2020] https://www.localhealth.org.uk/\#c=home).

322 Daily population movement data by MSOA was extracted from the COVID-19 Impact monitor

323 (https://www.oxford-covid-19.com/). Furthermore, rail passenger numbers and crowding statistics

324 (2018) were extracted for use a commuter density index by MSOA prior to the local down for use a

325 proxy for likely daily contacts with other individuals and mixing density (http://maps.dft.gov.uk/rail-

326 passengers-and-crowding/interactive-dashboard/index.html.

327 As a proxy for underlying chronic circulatory and respiratory disease burden, we also utilised

328 mortality rates from chronic obstructive pulmonary disease (COPD) from 2016-2018 by MSOA.

329 (Public Health England: Local Health. [Accessed: 24/08/2020]

330 https://www.localhealth.org.uk/\#c=home). 
For initial exploratory spatial analysis, we used the Getis-Ord Gi* statistic ( $\mathrm{Gi} *)^{20}$, also known as hotspot analysis, to identify significant higher and lower risk MSOA in terms of case and deaths rates for the observed input data period, namely week 9 to 34 . The significance level was set as 0.05 . A previously developed Bayesian space-time SEIR formulation ${ }^{9}$ was then applied to assess the spatiotemporal variability of COVID-19 transmission at small area scale (MSOA) and by week in England, by accounting for the modelled transmission dynamics of the pathogen, inherent spatialtemporal correlation in the data, and important contextual risk factors for both COVID-19 cases and deaths. We also included a sum of cases in shared neighbours in the preceding time point as an additional parameter in our model to further assess the dependence of caseloads in adjoined areas

${ }^{21}$. Based on the available data provided we the starting study week was denoted as week 9 of 2020 (starting 24 February 2020), with available case data at the time of extraction for this analysis available up to week 34 (starting 17 August 2020) and death data available up to week 26 (starting 22June 2020). We also limited the end date for the input data at week 34 to assess the predictive performance of the model into the future if we are to prove its predictive utility and thus use as a pre-emptive resource allocation/prioritization tool.

Let $y_{\mathrm{ij}}$ be weekly confirmed COVID-19 cases in MSOA region $i$ and week $j,(i=1, \ldots, 151 ; j=9, \ldots, 51)$. A discrete form SEIR model for the size of susceptible population at week $(j+1)$ and MSOA region $i$ is given by $S_{i, j+1}=S_{i, j}-I_{i, j}-R i_{j}$, where $S, I$ and $R$ represent the susceptible, infectious and removed (deaths and recovered) populations, respectively. The number of the infected COVID-19 cases in region $i$ and week $j$ were assumed to follow a Poisson distribution, with expected number of cases is a function of the serial interval or generation time as a time measure of disease communicability and defined as the number of infected cases in one median incubation period back $\left(j-1\right.$ week $\left.^{22}\right)$ i.e. and the susceptible population size in week $j$. The number of asymptomatic cases by week and MSOA is 
assumed to be a function of the observed case load in a given MSOA/week multiplied by a parameter for the additional fraction that are asymptomatic.

Hence, $\mathrm{l}_{\mathrm{ij}} \sim \operatorname{Pois}\left(\mu_{\mathrm{ij}}\right)$, and $\mu_{\mathrm{ij}}$ is given by ${ }^{9}$ :

$$
\mu_{\mathrm{ij}}=\beta_{\mathrm{ij}} \times S_{\mathrm{ij}} \times \mathrm{I}_{\mathrm{i}, \mathrm{j}-1}
$$

$S_{i j}=S_{i j-1}-I_{i j-1}-$ Asym $_{i j-1}-$ deaths $_{i j-1}$

$\log \left(\mu_{\mathrm{ij}}\right)=\log \left(\beta_{1 \mathrm{ij}}\right)+\log \left(S_{\mathrm{ij}}\right)+\log \left(\mathrm{l}_{\mathrm{i}, \mathrm{j}-1}\right)$ $\log \left(\beta_{1 \mathrm{ij}}\right)=b_{1} 0+b_{1} \times\left(\right.$ mobility $_{\mathrm{i}, \mathrm{j}}+b_{2} \times$ mobility $_{\mathrm{i}, \mathrm{j}} \times \ln$ (population density $\left.\mathrm{p}_{\mathrm{i}}\right)+\mathrm{u}_{1 \mathrm{i}}+\mathrm{v}_{1 \mathrm{i}}$ $\operatorname{Asym}_{\mathrm{ij}} \sim$ Pois $\left(\mathrm{U}_{\mathrm{ij}}{ }^{*}\right.$ asym) asym $\sim$ Uniform $(0.05,0.4)$

And deaths $s_{i j} \sim$ Pois $\left(\mathrm{dmu}_{\mathrm{ij}}\right)$, and $\mathrm{dmu}_{\mathrm{ij}}$ is dependent on caseloads 2 weeks prior ${ }^{23}$ and given by

$$
\begin{aligned}
& \log \left(\mathrm{dm} \mu_{\mathrm{ij}}\right)=\log \left(\beta_{2 \mathrm{ij}}\right)+\log \left(\mathrm{I}_{\mathrm{i}, \mathrm{j}-2}\right) \\
& \log \left(\beta_{2 \mathrm{ij}}\right)=b_{2} 0+b_{3} \times(\text { p70plus })_{\mathrm{i}}+b_{4} \times(\mathrm{e}-\mathrm{IMD})_{\mathrm{i}}+\mathrm{u}_{2 \mathrm{i}}+\mathrm{v}_{2 \mathrm{i}}
\end{aligned}
$$

Here $b=\left(b_{0}, b_{1}, b_{2}\right)$ is the vector of regression coefficients for the intercept (representing the logtransformed baseline transmission rate across all locations), mobility represents the observed weekly mobility by MSOA; mobility x population represents an interaction term to capture the varying impact of mobility change as a function of changing population density at MSOA level; p $_{70 p l u s}$ plus represents the proportion of the population in a given MSOA that were 70 years or older in 2020 in a given MSOA; e-IMD represents the proportion of the elderly people living in deprivation in a given MSOA in 2019; $u_{i}$ corresponds to structured (spatial) heterogeneity and represents spatial variation in transmission rate between regions that captures the effects of unobserved variables with an underlying spatial pattern; $v_{i}$ correspond to geographically unstructured (i.e. random effect) heterogeneity in the transmission rate; and $\mu_{i j}$ is the weekly transmission rate at MSOA $i$ and week $j$ after incorporating the spatiotemporal effects of local social-demographic factors, the 
transmission dynamics of COVID-19 outbreak (i.e. SEIR model) and random effects $\left(u_{i}\right.$ and $\left.v_{i}\right)$. All covariate coefficients had diffuse normal priors, given by $\boldsymbol{b} \sim N(0.0,1.0 \mathrm{E} 6)$. The variances of the random effects had uniform priors, $\sigma_{u} \sim U(0,5)$ and $\sigma_{v} \sim U(0,5)$.

A convolution CAR model ${ }^{24}$ was applied to decompose the log of area-level relative risks into the sum of two random effects, namely a structured spatial dependency effect and unstructured areal level heterogeneity effect through the random effects $u_{i}$ and $v_{i}$ respectively. The convolution approach has demonstrated good robust estimates across multiple studies and a range of disease clustering/modelling scenarios, for example ${ }^{25}$.This spatial structured effect models the effect of proximity using a first-order neighbourhood structure (i.e. MSOA adjacency matrix), whereby the random effect is assumed to have a normal distribution, with the conditional weighted mean given by the average of the neighbours.

We conducted various sensitivity analyses to estimate the impact of social distancing (as proxied by the daily population mobility covariate) on COVID-19 caseloads in England. The model coefficients for the mobility covariates from the full multivariable space-time model was applied to the baseline mobility prior to lockdown and propagated throughout the period assuming no change in mobility from baseline (that is, daily mobility patterns remained unchanged). Secondly, the observed rate of change of mobility at week 34 was propagated forward to week 51. Lastly, we assumed various fractions of mobility gain post week 34 and applied these to the difference between the counterfactual of no reduction in population movement and observed first wave outbreak dynamics under full lockdown to characterise the likely trajectory in England in the coming weeks.

A comparison of model fit was performed using the deviance information criterion (DIC). Posterior distributions for parameters of interest were obtained through Markov chain Monte Carlo (MCMC) sampling. Convergence was assessed by visual inspection of the trace/autocorrelation plots for the 

posteriors were less than $5 \%$ of the standard deviation for a given posterior. We ran 100,000 MCMC iterations and discarded the first 10,000 MCMC iterations as part of the model burn-in. We extracted

411 the mean point estimate for a given posterior as well as the $95 \%$ uncertainty intervals (2.5 to 97.5

412 percentile). This analysis was performed using WinBUGS version 1.4.3.

413

414 We validated the performance of the model using out of sample prediction by comparing the 415 predicted number of cases from the model post week 34 (observed case data truncation point) with 416 the observed cases to prove its utility as an early warning (pre-emptive) tool for resource allocation 417 and prioritization by MSOA resolution (Supplementary Material Section 3).

\section{Contributors}

420 BS and RP conceived and designed the study. AL assisted with adaptation of the statistical

421 framework. BS acquired and analysed the data. All authors interpreted the data. BS drafted the

422 manuscript. All authors critically revised the manuscript and approved the final version for

423 submission.

\section{Declaration of interests}

425 All authors declare no competing interests. 

insights.

\section{References}

$4301 \quad$ Lillie, P. J. et al. Novel coronavirus disease (Covid-19): the first two patients in the UK with person to person transmission. Journal of Infection 80, 578-606 (2020).

2 Hellewell, J. et al. Feasibility of controlling COVID-19 outbreaks by isolation of cases and contacts. The Lancet Global Health (2020).

3 Du, Z. et al. Risk for transportation of coronavirus disease from Wuhan to other cities in China. Emerging infectious diseases 26, 1049 (2020).

$4 \quad \mathrm{Wu}$, J. T., Leung, K. \& Leung, G. M. Nowcasting and forecasting the potential domestic and international spread of the 2019-nCoV outbreak originating in Wuhan, China: a modelling study. The Lancet 395, 689-697 (2020).

5 Bootsma, M. C. \& Ferguson, N. M. The effect of public health measures on the 1918 influenza pandemic in US cities. Proceedings of the National Academy of Sciences 104, 75887593 (2007).

6 Eggo, R. M., Cauchemez, S. \& Ferguson, N. M. Spatial dynamics of the 1918 influenza pandemic in England, Wales and the United States. Journal of the Royal Society Interface 8 , 233-243 (2011).

7 Fuhrmann, C. The effects of weather and climate on the seasonality of influenza: what we know and what we need to know. Geography Compass 4, 718-730 (2010).

8 Lawson, A. B. Statistical methods in spatial epidemiology. (John Wiley \& Sons, 2013).

9 Lawson, A. B. \& Song, H.-R. Bayesian hierarchical modeling of the dynamics of spatiotemporal influenza season outbreaks. Spatial and spatio-temporal epidemiology 1, 187-195 (2010).

10 Peto, J. et al. Universal weekly testing as the UK COVID-19 lockdown exit strategy. The Lancet 395, 1420-1421 (2020).

11 Kraemer, M. U. et al. The effect of human mobility and control measures on the COVID-19 epidemic in China. Science 368, 493-497 (2020).

12 Chinazzi, M. et al. The effect of travel restrictions on the spread of the 2019 novel coronavirus (COVID-19) outbreak. Science 368, 395-400 (2020).

13 Vollmer, M. A. et al. Report 20: Using mobility to estimate the transmission intensity of COVID-19 in Italy: A subnational analysis with future scenarios. (Technical Report May. Imperial College COVID-19 Response Team, 2020).

14 Prem, K. et al. The effect of control strategies to reduce social mixing on outcomes of the COVID-19 epidemic in Wuhan, China: a modelling study. The Lancet Public Health (2020).

15 Lau, H. et al. The positive impact of lockdown in Wuhan on containing the COVID-19 outbreak in China. Journal of travel medicine 27, taaa037 (2020).

$16 \mathrm{Ji}$, T. et al. Lockdown contained the spread of 2019 novel coronavirus disease in Huangshi city, China: Early epidemiological findings. Clinical Infectious Diseases (2020).

17 Chintalapudi, N., Battineni, G. \& Amenta, F. COVID-19 disease outbreak forecasting of registered and recovered cases after sixty day lockdown in Italy: A data driven model approach. Journal of Microbiology, Immunology and Infection (2020).

18 Matrajt, L. \& Leung, T. Evaluating the Effectiveness of Social Distancing Interventions to Delay or Flatten the Epidemic Curve of Coronavirus Disease. Emerging infectious diseases 26 (2020). 
47219 Stevens, G. A. et al. Guidelines for accurate and transparent health estimates reporting: the

473 GATHER statement. PLoS medicine 13, e1002056 (2016).

47420 Ord, J. K. \& Getis, A. Local spatial autocorrelation statistics: distributional issues and an

475 application. Geographical analysis 27, 286-306 (1995).

47621 Lawson, A. B., Rotejanaprasert, C., Moraga, P. \& Choi, J. A shared neighbor conditional

477 autoregressive model for small area spatial data. Environmetrics 26, 383-392 (2015).

47822 Lauer, S. A. et al. The incubation period of coronavirus disease 2019 (COVID-19) from

479

480 publicly reported confirmed cases: estimation and application. Annals of internal medicine 172, 577-582 (2020).

481

482

23 Baud, D. et al. Real estima

24 Besag, J., York, J. \& Mollié, A. Bayesian image restoration, with two applications in spatial statistics. Annals of the institute of statistical mathematics 43, 1-20 (1991). Best, N., Richardson, S. \& Thomson, A. A comparison of Bayesian spatial models for disease mapping. Statistical methods in medical research 14, 35-59 (2005). 


\section{Figures}
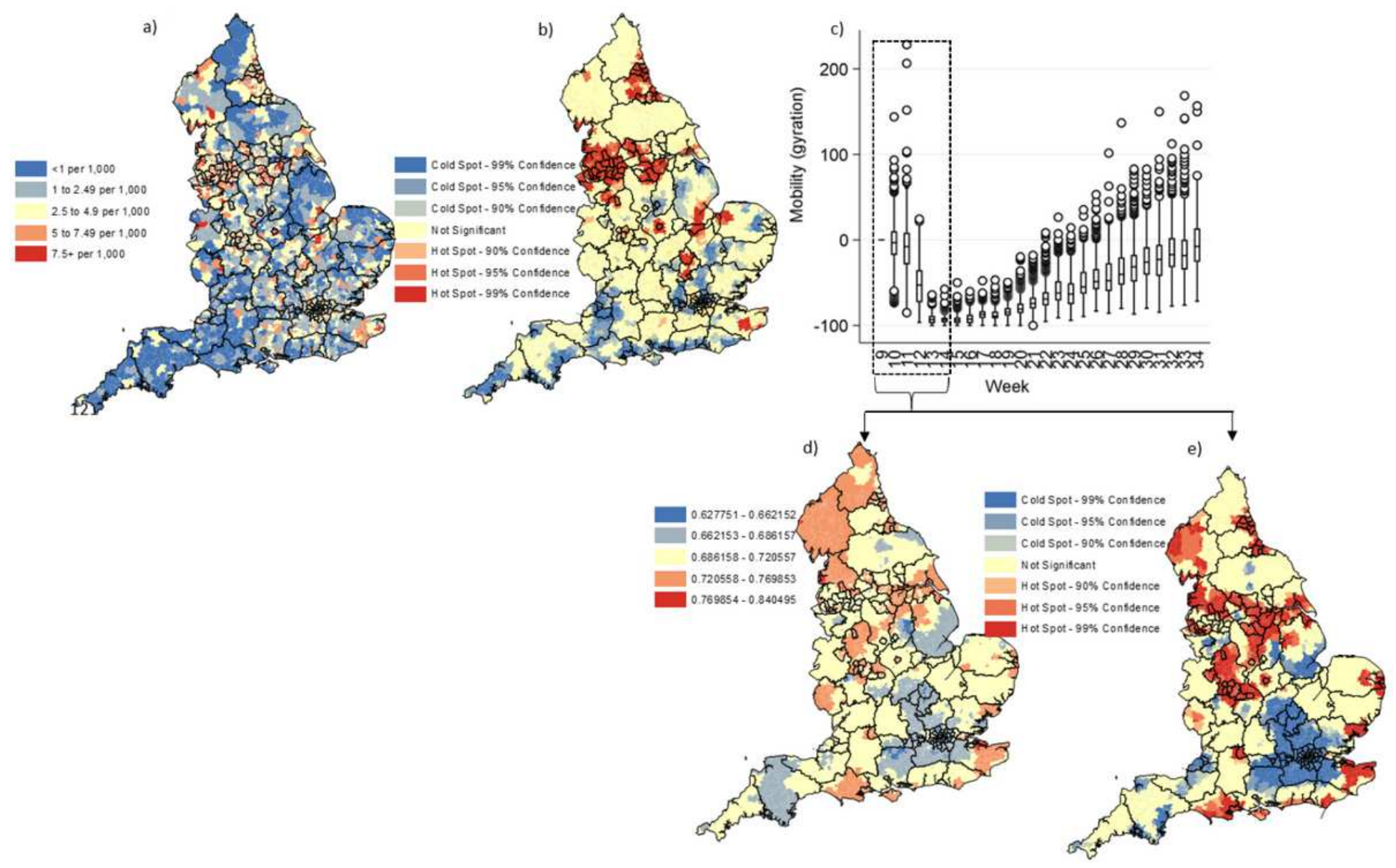

Figure 1

a) cumulative case rates at week 34 at English MSOA level ( $N=6791)$; b) statistically significant localised hot and cold spots for higher and lower cumulative COVID-19 cases at MSOA resolution at week 34 of 2020 using the Getis-Ord Gi* localised clustering statistic; c) Box plot of mobility by MSOA and week ; d) cumulative mobility loss (darker red indicates lower mobility loss) by MSOA in England ( $n=6791)$ from week 9 to 14 of 2020; e) statistically significant localised hot and cold spots for higher and lower cumulative mobility loss at MSOA resolution from week 9 to 14 of 2020 using the Getis-Ord Gi* statistic 


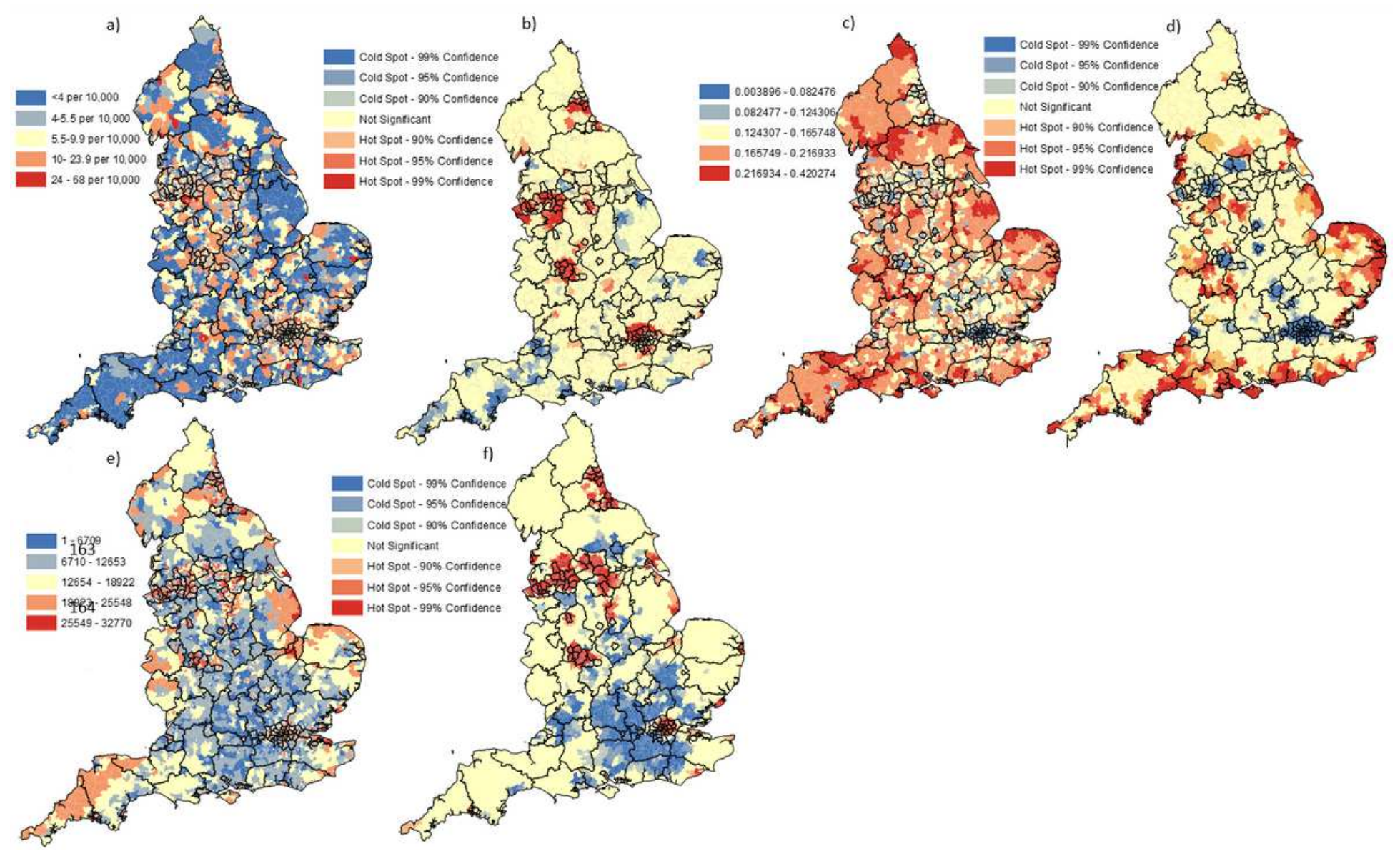

Figure 2

a) Cumulative case rates at week 34 at English MSOA level $(\mathrm{N}=6791)$; b) statistically significant localised hot and cold spots for higher and lower cumulative COVID-19 cases at MSOA resolution at week 34 of 2020 using the Getis-Ord Gi* statistic; c) proportion of population aged 70+ at MSOA resolution; d) statistically significant localised hot and cold spots for higher and lower proportion of population aged $70+$ at MSOA resolution using the Getis-Ord Gi* statistic; e) Index of multiple deprivation at MSOA resolution; f) statistically significant localised hot and cold spots for higher and lower proportion of IMD at MSOA resolution using the Getis-Ord Gi* statistic. 


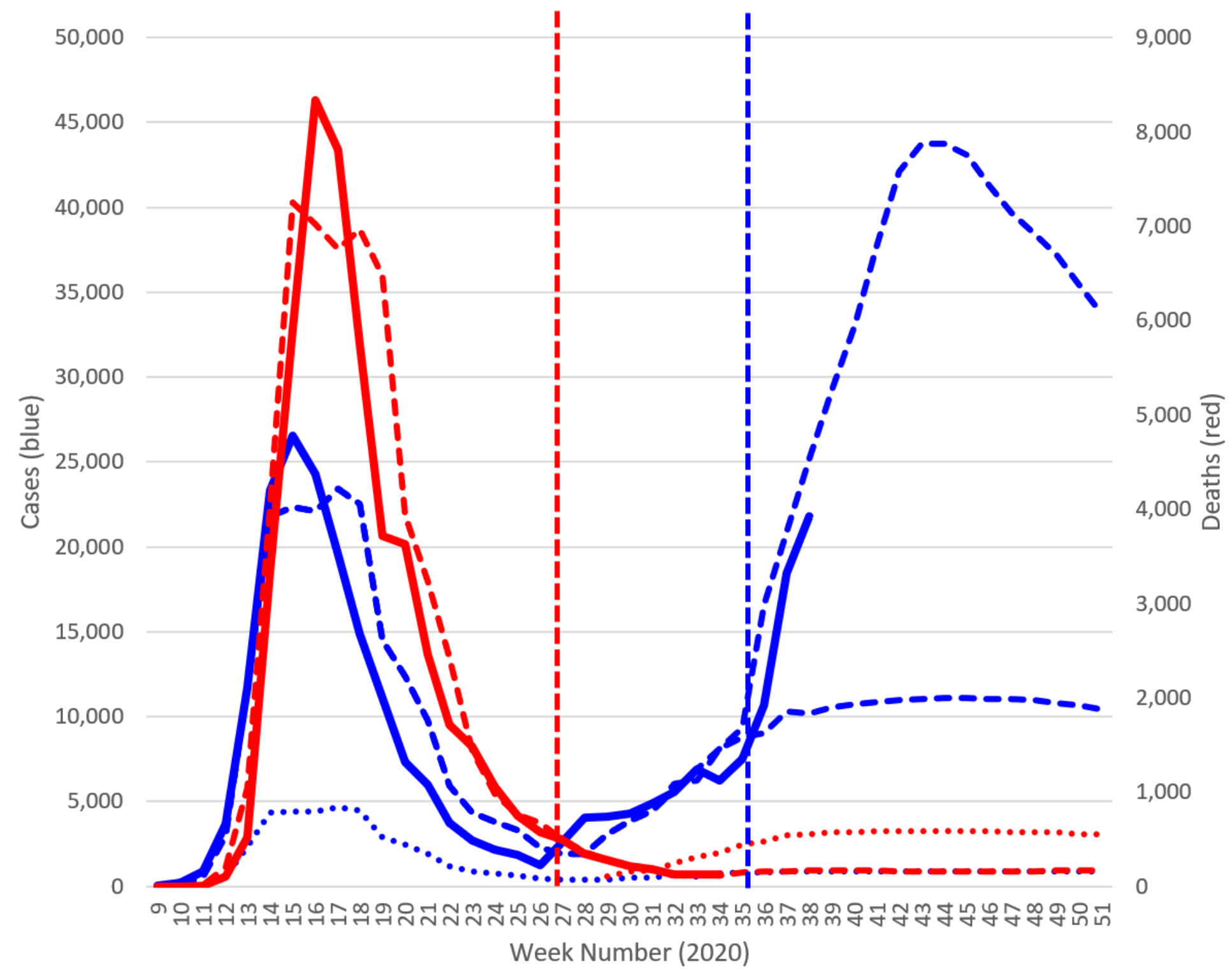

Figure 3

Observed and model fitted number of confirmed cases and deaths in England from week 9 to 34 of 2020, with projections to week 51 of 2020. Vertical coloured line represents the truncation point for observed input data for cases (week 34) and deaths (week 26) respectively. Blue solid line = observed number of weekly COVID-19 cases; blue dashed line = model fitted cases; dotted blue line = asymptomatic cases; red solid line = observed number of weekly COVID-19 deaths, red dashed line= model fitted deaths; red dotted line $=$ COVID-19 deaths based on mortality rate from first wave. 

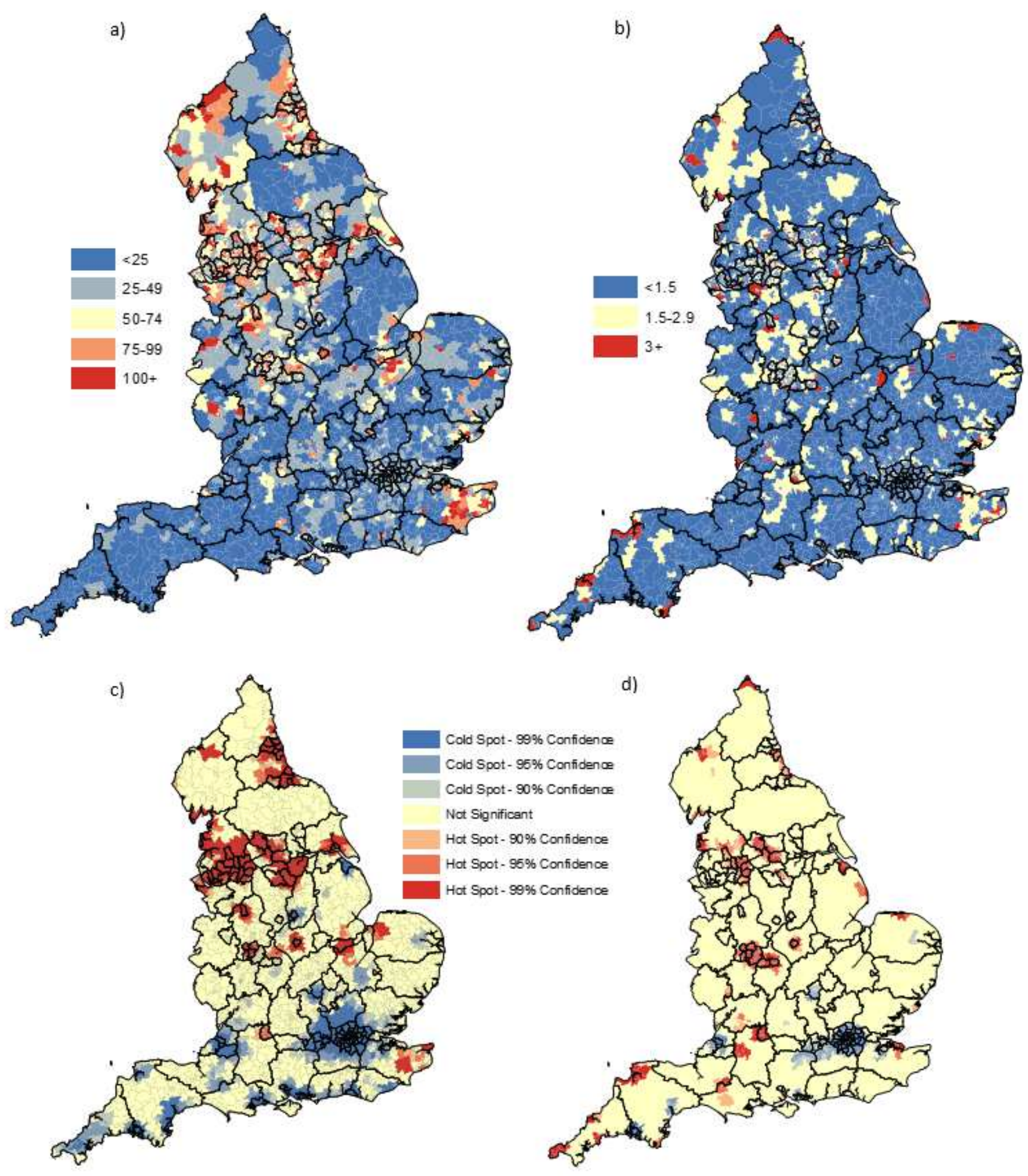

\section{Figure 4}

Model projected COVID case (a) and death (b) rates per 100,000 at MSOA level for week 34 to 40 of 2020; statistically significant localised hot and cold spots for COVID-19 case (c) and death (d) rates at MSOA level for week 34 to 40,2020

\section{Supplementary Files}


This is a list of supplementary files associated with this preprint. Click to download.

- SupplementaryMaterial.docx 